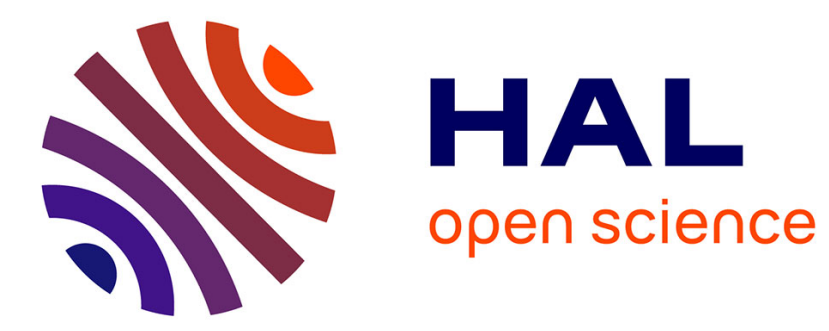

\title{
Une extension de la théorie de la multiplication complexe..
}

Jean-Pierre Wintenberger

\section{To cite this version:}

Jean-Pierre Wintenberger. Une extension de la théorie de la multiplication complexe... Journal für die reine und angewandte Mathematik, 2002, 552, pp.1-14. 10.1515/crll.2002.091 . hal-00129650

\section{HAL Id: hal-00129650 \\ https://hal.science/hal-00129650}

Submitted on 8 Feb 2007

HAL is a multi-disciplinary open access archive for the deposit and dissemination of scientific research documents, whether they are published or not. The documents may come from teaching and research institutions in France or abroad, or from public or private research centers.
L'archive ouverte pluridisciplinaire HAL, est destinée au dépôt et à la diffusion de documents scientifiques de niveau recherche, publiés ou non, émanant des établissements d'enseignement et de recherche français ou étrangers, des laboratoires publics ou privés. 


\title{
Une extension de la théorie de la multiplication complexe.
}

\author{
J.-P. Wintenberger
}

16.02.00.

Soit $p$ un nombre premier. Soit $K$ un corps de nombres ou une extension finie de $\mathbf{Q}_{p}$. Une clôture algébrique de $K$ étant donnée, soit $G_{K}$ le groupe de Galois absolu de $K$. Soit $H$ un groupe réductif connexe sur $\mathbf{Q}_{p}$, et $\rho: G_{K} \rightarrow H\left(\mathbf{Q}_{p}\right)$ une représentation $p$-adique. On suppose que $\rho$ est semi-stable ( en un idéal qui n'est pas au dessus de $p$, cela signifie que l'action de l'inertie est unipotente, en un idéal premier au dessus de $p$ au sens de [Fo94]). Soit $H^{\text {ab }}$ le quotient de $H$ par son sous-groupe $S$ des commutateurs. Soit $G_{K}^{\text {ab }}$ le plus grand quotient abélien de $G_{K}$. On sait comment décrire la représentation $p$-adique $\rho^{\mathrm{ab}}: G_{K}^{\mathrm{ab}} \rightarrow H^{\mathrm{ab}}\left(\mathbf{Q}_{p}\right)$, à l'aide des théories du corps de classes et de Hodge $p$-adique ([Se68], [Se78]). Soit $S_{\mathrm{sc}}$ le revêtement universel de $S$. On sait que le quotient de $H\left(\mathbf{Q}_{p}\right)$ par l'image de $S_{\mathrm{sc}}\left(\mathbf{Q}_{p}\right)$ est abélien ([De79],[Bo98]) ; notons le, comme dans [Bo98], $H_{\mathrm{ab}}^{0}\left(\mathbf{Q}_{p}, H\right)$. Notons $\rho \underline{\mathrm{ab}}$ le composé de $\rho$ avec $H\left(\mathbf{Q}_{p}\right) \rightarrow H_{\mathrm{ab}}^{0}\left(\mathbf{Q}_{p}, H\right)$. Nous généralisons la description de $\rho^{\mathrm{ab}}$ à $\rho \underline{\mathrm{ab}}$. Lorsque $K$ est un corps de nombres, et que $\rho$ est donnée par un motif pour des cycles de Hodge absolus compatibles au théorème de comparaison $p$-adique , nous décrivons $\rho$ ab à l'aide du groupe de Serre ([Se68]). Motivé par la conjecture de Lang qui dit que l'image de Galois dans la représentation adélique associée aux modules de Tate d'une variété abélienne contient un sous-groupe ouvert des homothéties, nous donnons des conditions suffisantes pour que l'image de $\rho \underline{\underline{a b}}$ contienne l'image des homothéties. Dans un article à venir, nous en déduirons des exemples de variétés abéliennes qui satisfont la conjecture de Lang.

Dans le 1.2., nous complétons les résultats de [Wi97] qui peuvent maintenant être démontrés dans leur généralité naturelle grâce au théorème de Colmez et Fontaine [CoFo99].

J'ai rédigé cet article alors que j'étais à l'IHES, à l'université de Berkeley et à l'université de Nottingham et je remercie ces instituts pour les excellentes conditions de travail qu'elles m'ont procurées.

\section{Rappels sur les groupes réductifs.}

Soit $E$ un corps de caractéristique 0 et $\bar{E}$ une clôture algébrique de $E$. Notons $G_{E}$ le groupe de Galois de $\bar{E} / E$. Soit $H$ un groupe réductif connexe sur $E$. Soit $(X, Y, R, \alpha \rightarrow$ $\left.\alpha^{\vee}, B\right)$ le système de racines épinglé de $H$ (cf par exemple [Se78]). Si $T$ est un tore maximal de $H_{\bar{E}}$, on a donc des isomorphismes de $X$ et $Y$ avec les groupes des caractères et groupes à un paramètre ( cocaractères ) de $T$ respectivement. Ces isomorphismes sont bien définis modulo l'action du groupe de Weyl $W(R)$. Le groupe de Galois $G_{E}$ agit de 
manière naturelle sur $X$ et $Y$, en laissant stables les systèmes de racines $R \subset X$ et $R^{\vee} \subset Y$, et la base $B$.

\subsection{Groupes à un paramètre.}

Soit $\Omega$ un corps algébriquement clos contenant $E$. Soit $\mu$ un groupe à un paramètre de $H_{\Omega}$, i.e. un morphisme du groupe multiplicatif $\mathbf{G}_{\mathrm{m}}$ dans $H$, défini sur $\Omega$. Comme l'image de $\mu$ est contenue dans un tore maximal de $H_{\Omega}$ et que tous les tores maximaux sont conjugués par automorphismes intérieurs, $\mu$ est conjugué à un groupe à un paramètre $\mu^{\prime}$ défini sur $\bar{E}$. Le groupe à un paramètre $\mu^{\prime}$ définit un élément de $Y$ modulo l'action du groupe de Weyl, et donc un élement de la chambre de $Y$ définie par $B$. On le note $\mathcal{C}(\mu)$. On a $\mathcal{C}\left(\mu_{1}\right)=\mathcal{C}\left(\mu_{2}\right)$ si et seulement si $\mu_{1}$ et $\mu_{2}$ sont conjugués par un automorphisme intérieur.

\subsection{Groupe fondamental de $H$ ([Bo98]).}

Soit $S$ le groupe des commutateurs de $H$, et $S_{\mathrm{sc}}$ le revêtement universel de $S$. Soit $\pi_{1}\left(H_{\bar{E}}\right)$ le groupe fondamental de $H_{\bar{E}}$ ([Bo98]). Si $T$ est un tore maximal de $H_{\bar{E}}$, et $T_{\mathrm{sc}}$ l'image réciproque de $T$ dans $\left(S_{\mathrm{sc}}\right)_{\bar{E}}$, on a donc :

$$
\pi_{1}\left(H_{\bar{E}}\right)=Y(T) / Y\left(T_{\mathrm{sc}}\right) .
$$

Ceci définit $\pi_{1}\left(H_{\bar{E}}\right)$ à isomorphisme unique près : si $\left(X, Y, R, \alpha \rightarrow \alpha^{\vee}, B\right)$ est le système de racines épinglé de $H, \pi_{1}\left(H_{\bar{E}}\right)$ s'identifie donc au quotient $Y / Q\left(R^{\vee}\right)$ de $Y$ par le réseau des poids radiciels du système de racines dual (i.e. le réseau engendré par les $\alpha^{\vee}$ ). En particulier, $\pi_{1}\left(H_{\bar{E}}\right)$ est muni d'une action naturelle de $G_{E}$. Le groupe fondamental de $H_{\bar{E}}$, au sens de Grothendieck, s'identifie à $\pi_{1}\left(H_{\bar{E}}\right) \otimes \widehat{\mathbf{Z}}(1)$. Le groupe de Weyl $W(R)$ agit trivialement sur $\pi_{1}\left(H_{\bar{E}}\right) \simeq Y / Q\left(R^{\vee}\right)$, puisque pour toute racine $\alpha^{\vee}$ de $R^{\vee}$, la réflexion $s_{\alpha^{\vee}}$ vérifie : $s_{\alpha^{\vee}}(y)-y=\langle\alpha, y\rangle \alpha^{\vee}$ pour tout $y \in Y$. Il en résulte une application de $W(R) \backslash Y$ dans $\pi_{1}\left(H_{\bar{E}}\right)$. Si $\mu$ est un groupe à un paramètre de $H_{\Omega}$ comme au 0.1 ., on note $\pi_{1}(\mu)$ l'image de $\mathcal{C}(\mu)$ dans $\pi_{1}\left(H_{\bar{E}}\right)$.

Soit $H^{\prime}$ un groupe réductif connexe sur $E$ et $H^{\prime} \rightarrow H$ une isogénie (centrale). Alors $\pi_{1}\left(H_{\bar{E}}^{\prime}\right)$ s'identifie à un sous-groupe d'indice fini de $\pi_{1}\left(H_{\bar{E}}\right)$ qui est stable sous l'action de $G_{E}$. Réciproquement, un tel sous-groupe provient de $H^{\prime} \rightarrow H, H^{\prime}$ étant bien défini à un isomorphisme au dessus de $H$ unique près. Si $\mu$ est un groupe à un paramètre de $H_{\Omega}, \mu$ se relève à $H^{\prime}$ si et seulement si $\pi_{1}(\mu)$ appartient à $\pi_{1}\left(H_{\bar{E}}^{\prime}\right)$. Si c'est le cas, le relèvement est unique.

\subsection{Le groupe $H_{\mathrm{ab}}^{0}(E, H)([\mathrm{Bo98}])$.}

M. Borovoi définit :

$$
H_{\mathrm{ab}}^{0}(E, H)=H^{0}\left(E, T_{\mathrm{sc}}(\bar{E}) \rightarrow T(\bar{E})\right),
$$

où $T_{\mathrm{sc}}(\bar{E})$ est placé en degré -1 et $T(\bar{E})$ en degré 0 , et $H^{0}(E,$.$) est l'hypercohomologie$ galoisienne. Cette définition ne dépend pas du choix de $T$ comme le montre l'isomorphisme 


$$
H_{\mathrm{ab}}^{\mathrm{O}}(E, H)=H^{0}\left(E, \pi_{1}\left(H_{\bar{E}}\right) \underset{\mathbf{Z}}{\otimes} \bar{E}^{*}\right)
$$

Pour $H$ et $H^{\prime}$ deux groupes réductifs connexes sur $E$ et un morphisme $g: \pi_{1}\left(H_{\bar{E}}\right) \rightarrow$ $\pi_{1}\left(H_{E}^{\prime}\right)$ commutant à l'action de $G_{E}$, on a donc un morphisme $H_{\mathrm{ab}}^{0}(E, H) \rightarrow H_{\mathrm{ab}}^{0}\left(E, H^{\prime}\right)$ : on le note $\mathrm{B}(g)$. Si $H^{\text {ab }}$ est le tore quotient de $H$ par $S$, on a une suite exacte :

$$
1 \rightarrow H^{1}\left(E, \operatorname{Ker}\left(S_{\mathrm{sc}}(\bar{E}) \rightarrow S(\bar{E})\right) \rightarrow H_{\mathrm{ab}}^{0}(E, H) \rightarrow H^{\mathrm{ab}}(E)\right.
$$

Les groupes $\operatorname{Ker}\left(S_{\mathrm{sc}}(\bar{E}) \rightarrow S(\bar{E})\right)$ et $\pi_{1}\left(S_{\bar{E}}\right)(1)$ s'identifient. On a un morphisme naturel : $\mathrm{ab}^{0}: H(E) \rightarrow H_{\mathrm{ab}}^{0}(E, H)$ dont le noyau est l'image de $S_{\mathrm{sc}}(E)$ dans $H(E)$. Si $E$ est un corps local non archimédien, le morphisme $H(E) \rightarrow H_{\mathrm{ab}}^{0}(E, H)$ est surjectif. On a donc dans ce cas une suite exacte :

$$
1 \rightarrow \operatorname{Im}\left(S_{\mathrm{sc}}(E) \rightarrow S(E)\right) \rightarrow H(E) \rightarrow H_{\mathrm{ab}}^{0}(E, H) \rightarrow 1
$$

\section{Etude locale.}

\subsection{Représentations $p$-adiques semi-stables à valeurs dans un groupe algébrique affine connexe.}

Soient $p$ un nombre premier et $\mathbf{Q}_{p}$ le corps des nombres $p$-adiques. Soit $K$ un corps de caractéristique 0 complet pour une valuation discrète à corps résiduel parfait $k$ de caractéristique $p$. Soit $\bar{K}$ une clôture algébrique de $K$ et $\overline{\mathbf{Q}_{p}}$ la clôture algébrique de $\mathbf{Q}_{p}$ contenue dans $\bar{K}$. Notons $G_{\mathbf{Q}_{p}}$ et $G_{K}$ les groupes de Galois de $\overline{\mathbf{Q}_{p}} / \mathbf{Q}_{p}$ et $\bar{K} / K$ respectivement.

Soit $H$ un groupe algébrique affine connexe sur $\mathbf{Q}_{p}$. Soit $\rho$ une représentation $p$-adique à valeurs dans $H$ i.e. un homomorphisme continu de $G_{K}$ dans $H\left(\mathbf{Q}_{p}\right)$, On suppose que $\rho$ est semi-stable i.e. que, pour toute représentation linéaire $\alpha: H \rightarrow \mathrm{GL}_{V}$ de $H$ dans un $\mathbf{Q}_{p}$-espace vectoriel $V$ de dimension finie, le composé $\alpha \circ \rho: G_{K} \rightarrow \mathrm{GL}_{\mathbf{Q}_{p}}(V)$ est semi-stable. Les propriétés tannakiennes de la catégorie des représentations semi-stables entraînent immédiatement qu'il suffit pour ceci que ce soit le cas pour une représentation linéaire fidèle $\alpha$ de $H$ ([Fo94]).

Soit $C_{p}$ la complétion de $\overline{\mathbf{Q}_{p}}$. La décomposition de Hodge-Tate des représentations $p$-adiques semi-stables composées de $\rho$ avec les représentations linéaires de $H$ définit un groupe à un paramètre $\mu_{\mathrm{HT}}:\left(\mathbf{G}_{\mathrm{m}}\right)_{C_{p}} \rightarrow H_{C_{p}}$ ([Se78] 1.4.). La classe de conjugaison (par automorphismes intérieurs) de $\mu_{\mathrm{HT}}$ est définie sur $K$ : ceci résulte de la formule : ${ }^{\tau} \mu_{\mathrm{HT}}=\operatorname{int}\left(\rho\left(\tau^{-1}\right)\right)\left(\mu_{\mathrm{HT}}\right)$, pour $\tau \in G_{K}$ ([Se78] 1.5.). Lorsque $H$ est réductif, il en résulte que $\mathcal{C}\left(\mu_{\mathrm{HT}}\right)$ et $\pi_{1}\left(\mu_{\mathrm{HT}}\right)$ sont fixes sous l'action de $G_{K}$.

\subsection{Relèvement des représentations semi-stables.}

1.2.1. Théorème. On suppose le corps résiduel $k$ algébriquement clos. Soient $H$ et $H^{\prime}$ deux groupes algébriques affines connexes sur $\mathbf{Q}_{p}$. Soit $f$ un morphisme surjectif de $H^{\prime}$ sur $H$ dont le noyau est un groupe de type multiplicatif (i.e. dont la composente neutre est un tore). Soit $\rho: G_{K} \rightarrow H\left(\mathbf{Q}_{p}\right)$ une représentation p-adique semi-stable. On 
suppose donné un groupe à un paramètre $\mu^{\prime}$ de $H^{\prime}$ défini sur $C_{p}$ tel que $f \circ \mu^{\prime}=\mu_{\mathrm{HT}}$ et tel que $\mathcal{C}\left(\mu^{\prime}\right)$ (cf 0.1.) soit fixe sous l'action de $G_{K}$. Alors, il existe une et une seule représentation p-adique semi-stable $\rho^{\prime}: G_{K} \rightarrow H^{\prime}\left(\mathbf{Q}_{p}\right)$, telle que : $f \circ \rho^{\prime}=\rho$ et que le groupe à un paramètre associé $\mu_{\mathrm{HT}}^{\prime}$ coïncide avec $\mu^{\prime}$.

1.2.2. Remarque. Soient $Y$ et $Y^{\prime}$ les groupes des cocaractères des systèmes de racines épinglés associés aux quotients de $H$ et $H^{\prime}$ par leurs radicaux unipotents. Se donner $\mu^{\prime}$ tel que $f \circ \mu^{\prime}=\mu_{\mathrm{HT}}$, revient à se donner un élément $y^{\prime}$ de $Y^{\prime}$ dont l'image par le morphisme $Y^{\prime} \rightarrow Y$ induit par $f$ coïncide avec $\mathcal{C}\left(\mu_{\mathrm{HT}}\right)$. Si un tel $y^{\prime}$ existe, son fixateur est un sousgroupe ouvert $G_{K^{\prime}}$ de $G_{K}$, et on peut appliquer le théorème après avoir remplacé $K$ par $K^{\prime}$. Si $f$ est une isogénie, $Y^{\prime}$ s'injecte dans $Y$, et on peut prendre $K^{\prime}=K$.

Démonstration.

1.2.3 Commenons par nous ramener au cas où $H$ et $H^{\prime}$ sont réductifs. Supposons le théorème vrai lorsque $H$ et $H^{\prime}$ sont réductifs. Soient $\operatorname{rad}_{\mathrm{u}}(H)$ et $\operatorname{rad}_{\mathrm{u}}\left(H^{\prime}\right)$ les radicaux unipotents de $H$ et $H^{\prime}$ respectivement et notons $H_{\text {red }}$ et $H_{\text {red }}^{\prime}$ les quotients de $H$ et $H^{\prime}$ par $\operatorname{rad}_{\mathbf{u}}(H)$ et $\operatorname{rad}_{\mathbf{u}}\left(H^{\prime}\right)$ respectivement. Puisque le noyau de $f$ est de type multiplicatif, il induit un isomorphisme de $\operatorname{rad}_{\mathrm{u}}(H) \operatorname{sur} \operatorname{rad}_{\mathrm{u}}\left(H^{\prime}\right)$. On applique le théorème à $H_{\text {red }}$ et $H_{\text {red }}^{\prime}$ et à la représentation $\rho_{\text {red }}$ de $G_{K}$ dans $H_{\text {red }}\left(\mathbf{Q}_{p}\right)$ obtenue en composant $\rho$ avec le morphisme $H\left(\mathbf{Q}_{p}\right) \rightarrow H_{\text {red }}\left(\mathbf{Q}_{p}\right)$. Il en résulte que $\rho_{\text {red }}$ se relève de manière unique en une représentation semi-stable $\rho_{\text {red }}^{\prime}: G_{K} \rightarrow H_{\text {red }}^{\prime}\left(\mathbf{Q}_{p}\right)$, dont le groupe à un paramètre de HodgeTate est le composé de $\mu^{\prime}$ avec le morphisme $H^{\prime} \rightarrow H_{\text {red }}^{\prime}$. Le groupe $H^{\prime}$ est le produit fibré de $H_{\text {red }}^{\prime}$ et de $H$ au dessus de $H_{\text {red }}$. On en déduit la représentation $\rho^{\prime}: G_{K} \rightarrow H^{\prime}\left(\mathbf{Q}_{p}\right)$. Le groupe $H^{\prime}$ possède une représentation linéaire fidèle qui est la somme directe d'une représentation linéaire de $H$ et d'une représentation linéaire de $H_{\text {red }}^{\prime}$. Comme la somme directe de deux représentation semi-stable l'est, on voit que $\rho^{\prime}$ est semi-stable. Le théorème s'en déduit alors immédiatement.

1.2.4. Supposons $H$ et $H^{\prime}$ réductifs. Soient $S$ et $S^{\prime}$ les groupes des commutateurs de $H$ et $H^{\prime}$ respectivement. Soient $H^{\mathrm{ab}}$ et $H^{\prime a b}$ les quotients de $H$ et $H^{\prime}$ par $S$ et $S^{\prime}$ respectivement. Notons $\rho^{\mathrm{ab}}$ le composé de $\rho$ avec $H\left(\mathbf{Q}_{p}\right) \rightarrow H^{\mathrm{ab}}\left(\mathbf{Q}_{p}\right)$. Construisons tout d'abord la représentation $p$-adique $\rho^{\prime \mathrm{ab}}$, composée de $\rho^{\prime}$ avec $H^{\prime}\left(\mathbf{Q}_{p}\right) \rightarrow H^{\prime \mathrm{ab}}\left(\mathbf{Q}_{p}\right)$.

Pour toute extension finie $E$ de $\mathbf{Q}_{p}$ contenue dans $\overline{\mathbf{Q}_{p}}$, notons $T_{E}$ le tore, restriction des scalaires à la Weil de $E$ à $\mathbf{Q}_{p}$ du groupe multiplicatif $\left(\mathbf{G}_{\mathrm{m}}\right)_{E}$. Le groupe de ses points à valeurs dans une $\mathbf{Q}_{p}$-algèbre $A$ est le groupe des éléments inversibles de $A \otimes \mathbf{Q}_{p} E$. En particulier, $T_{E}\left(\overline{\mathbf{Q}_{p}}\right) \simeq \Pi\left(\overline{\mathbf{Q}_{p}^{*}}\right)_{\tau}, \tau$ décrivant les différents $\mathbf{Q}_{p}$-plongements de $E$ dans $\overline{\mathbf{Q}_{p}}$. Le groupe $Y\left(T_{E}\right)$ des groupes à un paramètres de $\left(T_{E}\right) \overline{\mathbf{Q}_{p}}$ s'identifie au groupe abélien libre sur l'ensemble de ces plongements. On note $\mu_{E}$ le groupe à un paramètre de $\left(T_{E}\right) \overline{\mathbf{Q}_{p}}$ correspondant à l'inclusion de $E$ dans $\overline{\mathbf{Q}_{p}}$. Soit $E_{\mathrm{nr}}$ l'extension maximale non ramifiée de $E$ contenue dans $\overline{\mathbf{Q}_{p}}$. On sait que les groupes formels de Lubin et Tate associés aux différentes uniformisantes de $E$ définissent une représentation $p$-adique cristalline du groupe de Galois $G_{E_{\mathrm{nr}}}$ à valeurs dans $T_{E}\left(\mathbf{Q}_{p}\right)$, dont le groupe à un paramètre de Hodge-Tate est $\mu_{E}$ ([Se78] 2). Composant avec le morphisme naturel $G_{K} \rightarrow G_{E}$, on obtient une représentation $p$-adique de $G_{K}$ que l'on note $\rho_{E}$.

Le groupe à un paramètre $\mu_{\mathrm{HT}}^{\mathrm{ab}}$ composé de $\mu_{\mathrm{HT}}$ et de $H \rightarrow H^{\mathrm{ab}}$ est défini sur une extension finie $E$ de $\mathbf{Q}_{p}$ contenue dans $K$ (1.1.). Il existe un unique morphisme $T_{E} \rightarrow H^{\mathrm{ab}}$ 
défini sur $\mathbf{Q}_{p}$ et qui envoie $\mu_{E}$ sur $\mu_{\mathrm{HT}}^{\mathrm{ab}}$. La représentation $p$-adique $\rho^{\mathrm{ab}}$ est le composé de $\rho_{E}$ avec ce morphisme : on peut le voir d'abord après restriction à une extension finie de $K$ grâce à [Se78] loc. cit. , puis conclure car deux représentations semi-stables de $G_{K}$ qui coïncident sur un sous-groupe ouvert coïncident (c'est évident que leurs $(\phi, N)$-modules filtrés coïncident).

Notons $\mu^{\text {ab }}$ le composé de $\mu^{\prime}$ et de $H^{\prime} \rightarrow H^{\prime a b}$. Le groupe à un paramètre $\mu^{\prime a b}$ est défini sur une extension finie $E^{\prime}$ de $\mathbf{Q}_{p}$ contenue dans $\overline{\mathbf{Q}_{p}}$. Puisque par hypothèse $\mathcal{C}\left(\mu^{\prime}\right)$ est fixe sous l'action de $G_{K}$, on peut supposer que $E^{\prime}$ est contenu dans $K$. On peut de plus supposer que $E^{\prime}$ contient $E$. Le groupe à un paramètre $\mu^{\prime a b}$ définit un morphisme $T_{E^{\prime}} \rightarrow H^{\prime \mathrm{ab}}$. Composé avec $\rho_{E^{\prime}}$, on obtient la représentation $\rho^{\mathrm{ab}}$.

1.2.5. Le morphisme naturel $H^{\prime} \rightarrow H \times{ }_{H^{\text {ab }}} H^{\prime a b}$ est une isogénie. On voit donc que l'on est ramené au cas où $H^{\prime} \rightarrow H$ est une isogénie, ce que nous supposerons désormais. Le corps résiduel $k$ étant algébriquement clos, l'adhérence de Zariski de l'image de $\rho$ est un groupe connexe, car une repésentation $p$-adique semi-stable de $G_{K}$ à image finie est triviale. On peut remplacer $H$ par cette adhérence de Zariski et $H^{\prime}$ par la composante neutre de l'image réciproque de $H$ dans $H^{\prime}$, et supposer que l'image de $\rho$ est Zariski dense dans $H$, ce que nous supposerons désormais (cependant $H$ et $H^{\prime}$ ne sont plus nécessairement des groupes réductifs).

Notons $\operatorname{Rep}_{\mathbf{Q}_{p}}(H)$ la catégorie des représentations linéaires de $H$ dans les $\mathbf{Q}_{p^{-}}$espaces vectoriels de dimensions finies et soit $\alpha_{0}$ un objet de $\operatorname{Rep}_{\mathbf{Q}_{p}}(H)$ qui est une représentation linéaire fidèle de $H$. Comme l'image de $\rho$ est Zariski dense dans $H$, si à $\alpha$ représentation linéaire de $H$, on associe $\alpha \circ \rho$, on définit une équivalence de la catégorie tannakienne $\operatorname{Rep}_{\mathbf{Q}_{p}}(H)$ avec la sous-catégorie tannakienne de celle des repésentations semi-stables de $G_{K}$ engendrée par $\alpha_{0} \circ \rho$. Soit $K_{0} \subset K$ le corps des fractions des vecteurs de Witt à coefficients dans $k$. Pour $\alpha: H \rightarrow \mathrm{GL}_{V}$ de $\operatorname{Rep}_{\mathbf{Q}_{p}}(H)$, soit $D(\alpha)$ le $(\phi, N)$-module filtré associé à $\alpha \circ \rho$ par le foncteur de Fontaine ([Fo94]). Posons $D_{0}=D\left(\alpha_{0}\right)$. Si à $\alpha$, on associe le $K_{0}$-espace vectoriel sous-jacent à $D(\alpha)$, on définit un foncteur fibre de $\operatorname{Rep}_{\mathbf{Q}_{p}}(H)$ à valeurs dans $K_{0}$. Soit $H_{D_{O}}$ le groupe de ses $\otimes$-automorphismes : le groupe algébrique $H_{D_{0}}$ s'identifie donc au groupe des $\otimes$-automorphismes du foncteur fibre espace vectoriel sous-jacent de la sous-catégorie tannakienne de celle des $(\phi, N)$-modules filtrés admissibles engendrée par $D_{0}(c f[\mathrm{Wi} 97])$. Soit $I s$ le $H$-torseur des $\otimes$-isomorphismes entre le foncteur fibre $\mathbf{Q}_{p}$-espace vectoriel sous-jacent de $\operatorname{Rep}_{\mathbf{Q}_{p}}(H)$, tensorisé avec $K_{0}$, et le foncteur fibre $\alpha \mapsto D(\alpha)$. Comme $H$ est connexe, on sait, grâce à un théorème de Steinberg ([St65]), que $i$ a un point à valeurs dans $K_{0}$. Soit $i$ un tel point. Soit $V_{0}$ le $\mathbf{Q}_{p}$-espace vectoriel sous-jacent à $\alpha_{0}$. Le point $i$ définit donc un isomorphisme de $K_{0} \otimes \mathbf{Q}_{p} V_{0}$ sur $D_{0}$. Si $\sigma$ est le Frobenius de $K_{0}$, soit $c$ lélément de $H_{D_{0}}\left(K_{0}\right)$ défini par : $\phi_{D_{0}} \circ c^{-1}=i \circ(\sigma \otimes \mathrm{id}) \circ i^{-1}$. Il est clair que $\left(D_{0}, \phi_{D_{0}} \circ c^{-1}\right)$ est un isocristal de pente 0 et donc, avec les notations de [Wi97] 1.6. , $c$ est un élément $C_{D_{0}, 0}$. Soit, comme dans [Wi97], $H_{D_{0}, c}$ le groupe algébrique sur $\mathbf{Q}_{p}$ qui est défini à partir de $H_{D_{0}}\left(K_{0}\right)$ par la donnée de descente définie par $\phi_{D_{0}} \circ c^{-1}$. Le point $i \in I s\left(K_{0}\right)$ définit un isomorphisme de $H$ sur $H_{D_{0}, c}$, défini sur $K_{0}$; on le note $\underline{i}$. Les différents choix de $i$ dans $I s\left(K_{0}\right)$ définissent $\underline{i}$ modulo automorphismes intérieurs par les éléments de $H\left(K_{0}\right)$ (ou de $H_{D_{0}}\left(K_{0}\right)$ ). Il en résulte un isomorphisme bien défini de $\pi_{1}(H)$ sur $\pi_{1}\left(H_{D_{0}, c}\right)$, qui commute aux actions du groupe de Galois $G_{\mathbf{Q}_{p}}$. On a donc sur $\pi_{1}\left(H_{D_{0}}\right)$ une action de $G_{\mathbf{Q}_{p}}$ (qui ne dépend pas du choix de $c \in C_{D_{0}, 0}, c f[$ Wi97] 2.2.). Soit 
$\tilde{H}$ le groupe réductif connexe sur $K_{0}$ et l'isogénie $\tilde{H} \rightarrow H_{D_{0}}$ correspondant au sous groupe $\pi_{1}\left(H^{\prime}\right)$ de $\pi_{1}(H)$. La classe de conjugaison de $\mu_{\mathrm{HT}}^{-1}$ correspond, via $\underline{i}$, à celle donnée par un $\otimes$-scindage $\mu^{\prime}$ de la filtration dans la catégorie tannakienne des $(\phi, N)$-modules filtrés ([Wi88]). L'isogénie $\tilde{H} \rightarrow H_{D_{0}}$ vérifie les deux conditions du théorème 2.2.2. de [Wi97] : $\pi_{1}(\hat{H})$ est stable sous l'action naturelle de $G_{\mathbf{Q}_{p}}$ et $\mu^{\prime}$ se relève. Il existe donc un $(\phi, N)$-module filtré faiblement admissible $D^{\prime}$, tel que la sous-catégorie tannakienne qu'il engendre contienne $D_{0}$, et que le morphisme $H_{D^{\prime}} \rightarrow H_{D_{0}}$ soit isomorphe, au dessus de $H$, à l'isogénie $\tilde{H} \rightarrow H_{D_{0}}$. Grâce au théorème de P. Colmez et J.-M. Fontaine ([CoFo99]), le module filtré $D^{\prime}$ est admissible. Il lui correspond une représentation $p$-adique semi-stable $\rho^{\prime}: G_{K} \rightarrow \mathrm{GL}_{\mathbf{Q}_{p}}\left(V^{\prime}\right)$, telle que, si $H^{\prime \prime}$ est l'adhérence de Zariski de l'image de $\rho^{\prime}$, l'isogénie $H^{\prime \prime} \rightarrow H$ corresponde au sous-groupe $\pi_{1}\left(H^{\prime}\right)$ de $\pi_{1}(H)$. Il en résulte un isomorphisme au dessus de $H$ entre $H^{\prime \prime}$ et $H^{\prime}$. La représentation $p$-adique $G_{K} \rightarrow H^{\prime \prime}\left(\mathbf{Q}_{p}\right) \simeq H^{\prime}\left(\mathbf{Q}_{p}\right)$ convient et cela achève de prouver le théorème.

On a le corollaire suivant, qui ne suppose pas le corps résiduel algébriquement clos, et qui est cité dans [CoFo99] :

1.2.6. Corollaire. Soit $f: H^{\prime} \rightarrow H$ une isogénie de groupes algébriques affines définis sur $\mathbf{Q}_{p}$, soient $\rho^{\prime}: G_{K} \rightarrow H^{\prime}\left(\mathbf{Q}_{p}\right)$ un homomorphisme continu et $\rho=f_{\mathbf{Q}_{p}} \circ \rho^{\prime}$ : $G_{K} \rightarrow H\left(\mathbf{Q}_{p}\right)$. Si $\rho$ est semi-stable et $\rho^{\prime}$ de Hodge-Tate, alors $\rho^{\prime}$ est potentiellement semi-stable.

Démonstration. Puisqu'une représentation $p$-adique d'un corps local est semi-stable si et seulement si sa restriction à l'inertie l'est, on peut supposer le corps résiduel algébriquement clos. Le groupe à un paramètre $\mu_{\mathrm{HT}}^{\prime}$ associé à $\rho^{\prime}$ est un relèment de $\mu_{\mathrm{HT}}$ à $H^{\prime}$. Comme $f$ est une isogénie, $\mathcal{C}\left(\mu_{\mathrm{HT}}^{\prime}\right)$ est fixe sous l'action de $G_{K}$ (1.1. et 1.2.2.). Le théorème donne donc l'existence d'un relèvement semi-stable $\rho^{\prime \prime}$ de $\rho$ à $H^{\prime}$. Les représentations $\rho^{\prime}$ et $\rho^{\prime \prime}$ diffèrent par un caractères de $G_{K}$ à valeurs dans le noyau de $f_{\mathbf{Q}_{p}}$, donc coïncident sur un sous-groupe ouvert de $G_{K}$. Le corollaire en résulte.

1.2.7. Corollaire. On reprend les hypothèses du théorème sauf que l'on suppose le corps résiduel fini et non pas algébriquement clos. Alors, il existe une extension finie non ramifiée $K^{\prime}$ de $K$ et une représentation p-adique semi-stable $\rho^{\prime}: G_{K^{\prime}} \rightarrow H^{\prime}\left(\mathbf{Q}_{p}\right)$, telle que : $f \circ \rho^{\prime}=\rho$ et que le groupe à un paramètre associé $\mu_{\mathrm{HT}}^{\prime}$ coïncide avec $\mu^{\prime}$.

Démonstration. Soit $q$ le cardinal de $k$ et $F$ un élément de $G_{K}$ dont l'image dans $\operatorname{Gal}(\bar{k} / k)$ est $x \mapsto x^{q}$. Comme $\mathrm{H}^{1}\left(\mathbf{Q}_{p}, \operatorname{Ker}(f)\left(\overline{\mathbf{Q}_{p}}\right)\right)$ est un groupe fini ([CG] chap. 2 5.8.), une puissance finie $F^{a}$ de $F$ se relève à $H^{\prime}\left(\mathbf{Q}_{p}\right)$. On peut prendre pour $K^{\prime}$ l'extension non ramifiée de degré $a$ de $K$ : c'est clair si $H$ est abélien et le cas où $f$ est une isogénie résulte comme dans le 1.2. de [Wi95] de l'unicité du relèvement de la restriction de $\rho$ à l'inertie.

\subsection{Description de la restriction à l'inertie du composé $\rho^{\text {ab }}$ de} $\rho: G_{K} \rightarrow H\left(\mathbf{Q}_{p}\right)$ et de $\mathrm{ab}^{0}: H\left(\mathbf{Q}_{p}\right) \rightarrow H_{\mathrm{ab}}^{0}\left(\mathbf{Q}_{p}, H\right)$.

On suppose le corps résiduel fini. Soit $H$ un groupe réductif connexe sur $\mathbf{Q}_{p}$ et soit $\rho: G_{K} \rightarrow H\left(\mathbf{Q}_{p}\right)$ une représentation $p$-adique semi-stable ( $c f$ 1.1.). Soit, comme dans 1.2.4., $T_{K}$ le tore restriction des scalaires à la Weil de $K$ à $\mathbf{Q}_{p}$ du groupe multiplicatif sur $K$. Comme $\pi_{1}\left(\mu_{\mathrm{HT}}\right)$ est fixe sous l'action de $G_{K}$, on voit que l'on a un unique morphisme 
$g$ commutant à l'action de $G_{\mathbf{Q}_{p}}$ de $Y\left(T_{K}\right)$ dans $\pi_{1}\left(H_{\overline{\mathbf{Q}_{p}}}\right)$ qui envoie $\mu_{K}$ sur $\pi_{1}\left(\mu_{\mathrm{HT}}\right)$. On en déduit le morphisme $\mathrm{B}(g)(c f$ 0.3.) :

$$
K^{*} \simeq T_{K}\left(\mathbf{Q}_{p}\right) \simeq H_{\mathrm{ab}}^{0}\left(\mathbf{Q}_{p}, T_{K}\right) \rightarrow H_{\mathrm{ab}}^{0}\left(\mathbf{Q}_{p}, H\right)
$$

Soit $K^{\mathrm{ab}}$ l'extension maximale abélienne de $K$ contenue dans $\overline{\mathbf{Q}_{p}}$, et désignons par $\mathrm{I}\left(K^{\mathrm{ab}} / K\right)$ le sous-groupe d'inertie de l'extension $K^{\mathrm{ab}} / K$. Le composé de $\rho: G_{K} \rightarrow H\left(\mathbf{Q}_{p}\right)$ avec $\mathrm{ab}^{0}: H\left(\mathbf{Q}_{p}\right) \rightarrow H_{\mathrm{ab}}^{\mathrm{o}}\left(\mathbf{Q}_{p}, H\right)$, se factorise à travers le groupe de Galois $\operatorname{Gal}\left(K^{\mathrm{ab}} / K\right)$.

L'application de réciprocité définit un isomorphisme $r$ du groupe des unités $U_{K}$ de $K$ sur $\mathrm{I}\left(K^{\mathrm{ab}} / K\right)$. Notons encore $\rho \underline{\mathrm{ab}}: \mathrm{I}\left(K^{\mathrm{ab}} / K\right) \rightarrow H_{\mathrm{ab}}^{0}\left(\mathbf{Q}_{p}, H\right)$ la restriction à $\mathrm{I}\left(K^{\mathrm{ab}} / K\right)$ du composé de $\rho$ et de $H\left(\mathbf{Q}_{p}\right) \rightarrow H_{\mathrm{ab}}^{\mathrm{o}}\left(\mathbf{Q}_{p}, H\right)$

Proposition. Le composé $\rho \underline{\mathrm{ab}} \circ \mathrm{r}$ de $\rho \underline{\mathrm{ab}}$ et de l'isomorphisme de réciprocité coïncide, au signe près, avec $\mathrm{B}(g):$ pour $u \in U_{K}$, on $a: \rho \underline{\mathrm{ab}} \circ r(u)=\mathrm{B}(g)\left(u^{-1}\right)$.

Démonstration. C'est démontré dans le 2 de [Se78] si $H$ est un tore. C'est donc prouvé dans le cas où le groupe des commutateurs de $H$ est simplement connexe. Dans le cas général, il résulte de la proposition 3.2.1. de [Wi97] qu'il existe $H^{\prime}, f$ et $\mu^{\prime}$ vérifiant les hypothèses du corollaire 1.2.7., avec le groupe des commutateurs de $H^{\prime}$ simplement connexe. Il existe donc une extension finie non ramifiée $K^{\prime}$ de $K$ et une représentation semi-stable $\rho^{\prime}: G_{K^{\prime}} \rightarrow H^{\prime}\left(\mathbf{Q}_{p}\right)$ telle que $f \circ \rho^{\prime}=\rho$. On connait la proposition pour $\rho^{\prime}$. On la déduit facilement pour $\rho$ à l'aide du diagramme commutatif suivant :

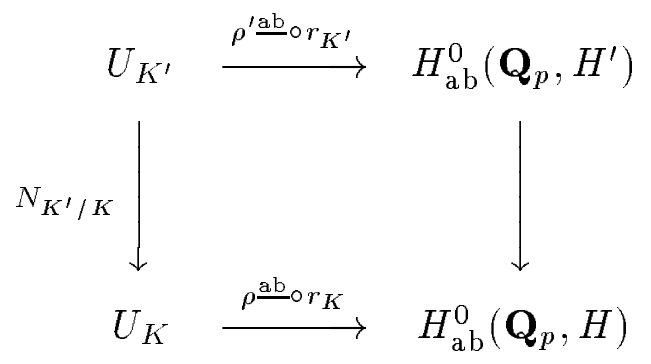

et de la surjectivité de la norme $N_{K^{\prime} / K}: U_{K^{\prime}} \rightarrow U_{K}$, puisque $K^{\prime} / K$ est non ramifiée.

Exemples. Soit $V$ un $\mathbf{Q}_{p}$-espace vectoriel de dimension finie et $q$ une forme quadratique non dégénérée sur $V$, de discriminant $\operatorname{disc}(q)$.

1) Soit $\rho: G_{K} \rightarrow \operatorname{SO}(q)\left(\mathbf{Q}_{p}\right)$ une représentation semi-stable. Soit $\theta: \operatorname{SO}(q)\left(\mathbf{Q}_{p}\right) \rightarrow$ $\mathbf{Q}_{p}^{*} / \mathbf{Q}_{p}^{* 2}$ la norme spinorielle $i . e$. le cobord $\mathrm{SO}(q)\left(\mathbf{Q}_{p}\right) \rightarrow H^{1}\left(\mathbf{Q}_{p}, \mu_{2}\right)$ pour la suite exacte

$$
1 \rightarrow \mu_{2} \rightarrow \operatorname{Spin}(q) \rightarrow \mathrm{SO}(q) \rightarrow 1
$$

On voit facilement que la proposition entraîne que pour $\sigma$ dans le sous-groupe d'inertie

$$
\theta(\rho(\sigma))=\chi^{a}(\sigma) \bmod . \mathbf{Q}_{p}^{* 2}
$$

où $\chi$ est le caractère cyclotomique, $a$ l'entier $\sum h_{i}$, les $h_{i}$ étant les nombres de Hodge i.e. les entiers $\operatorname{dim}_{K}\left(\operatorname{gr}^{i}\left(D_{K}(V)\right)\right.$, la somme portant sur les entiers positifs impairs (ou 
négatifs impairs, on a $\left.h_{i}=h_{-i}\right)$. On généralise ainsi à toutes les représentations semistables la formule $2 \mathrm{du}$ th. 2 de T. Saito [Sa95].

2) Supposons la dimension $d$ de $V$ paire. Soit $\operatorname{Sim}^{+}(q)$ le groupe des similitudes directes. Soit $\rho: G_{K} \rightarrow \operatorname{Sim}^{+}(q)\left(\mathbf{Q}_{p}\right)$ une représentation provenant d'un groupe $p$-divisible de dimension $d / 2$ (et de hauteur $d$ ). Alors Fil $^{1} D_{K}$ est un sous-espace totalement isotrope pour $q$. Décrivons la restriction de $\rho$ ab à l'inertie si $q$ est d'indice maximal $d / 2$ ou si $(-1)^{d / 2} \operatorname{disc}(q)$ n'est pas un carré dans $\mathbf{Q}_{p}$. On pourrait faire une description similaire mais d'énoncé plus compliqué si $(-1)^{d / 2} \operatorname{disc}(q)$ est un carré dans $\mathbf{Q}_{p}$ et $q$ n'est pas d'indice maximal.

Si $q$ est la forme d'indice $d / 2$, soit $W$ un sous-espace totalement isotrope de $V$ de dimension $d / 2$ dans la même orbite sous $\mathrm{SO}(q)$ que le sous-espace totalement isotrope de poids 1 pour la décomposition de Hodge-Tate et $W^{\prime}$ un supplémentaire de $W$ qui est totalement isotrope. Soit $\eta: \mathbf{Q}_{p}^{*} \rightarrow \operatorname{Sim}^{+}(q)\left(\mathbf{Q}_{p}\right)$ le groupe à un paramètre qui à $W$ comme sous-espace de poids 1 et $W^{\prime}$ comme sous-espace de dimension 0 et $\eta \underline{\text { ab }}$ le composé de $\eta$

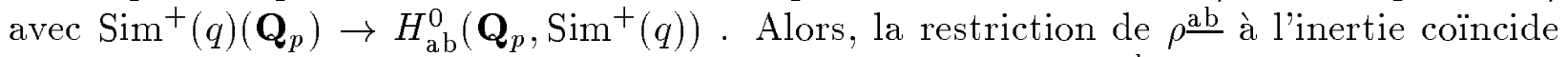
avec le composé du caractère cyclotomique $\chi: G_{K} \rightarrow \mathbf{Q}_{p}^{*}$ avec $\eta \underline{\text { ab }}$.

Supposons que $(-1)^{d / 2} \operatorname{disc}(q)$ n'est pas un carré dans $\mathbf{Q}_{p}$ et soit $K_{1}$ l'extension quadratique de $\mathbf{Q}_{p}$ engendrée par la racine carrée de $(-1)^{d / 2} \operatorname{disc}(q)$. Alors, comme la classe de conjugaison de $\mu_{\mathrm{HT}}$ est définie sur $K, K_{1}$ est inclus dans $K$. Soit $\chi_{1}: I_{K} \rightarrow K_{1}^{*}$ le caractère du sous-groupe d'inertie de $G_{K}$ donnant l'action sur de $I_{K}$ sur le module de Tate d'un groupe formel de Lubin-Tate pour $K_{1}$. Alors $V$ se décompose en la somme orthogonale d'un $\mathbf{Q}_{p}$-espace vectoriel $W \oplus W^{\prime}$ de dimension $d-2$ qui est hyperbolique, $W$ et $W^{\prime}$ totalement isotropes, et d'un $\mathbf{Q}_{p}$-espace vectoriel $V_{1}$ de dimension 2 . Le $K_{1}$-espace vectoriel $K_{1} \otimes \mathbf{Q}_{p} V_{1}$ est hyperbolique, et donc somme directe de deux sous-espaces vectoriels isotropes de dimension 1. Soit $W_{1}$ celui des deux tel que $W_{1} \oplus W$ soit dans la même orbite sous $\operatorname{SO}(q)$ que le sous-espace de poids 1 pour la décomposition de Hodge-Tate et $W_{1}^{\prime}$ l'autre. L'application $V_{1} \rightarrow K_{1} \otimes_{\mathbf{Q}_{p}} V_{1}$ composé avec la projection sur $W_{1}$ de noyau $W_{1}^{\prime}$ est un isomorphisme ce qui permet de munir $V_{1}$ d'une structure de $K_{1}$-espace vectoriel. Faisons agir $\lambda \in K_{1}^{*}$ sur $V$ par multiplication sur $V_{1}$ pour cette structure de $K_{1}$-espace vectoriel, par multiplication par $N_{K_{1} / \mathbf{Q}_{p}}(\lambda)$ sur $W$ et par l'identité sur $W^{\prime}$. On définit ainsi $\eta_{1}: K_{1}^{*} \rightarrow \operatorname{Sim}^{+}(q)\left(\mathbf{Q}_{p}\right)$. Notons $\eta_{1}^{a b}$ le composé de $\eta_{1}$ avec $\operatorname{Sim}^{+}(q)\left(\mathbf{Q}_{p}\right) \rightarrow H_{\mathrm{ab}}^{0}\left(\mathbf{Q}_{p}, \operatorname{Sim}^{+}(q)\right)$. Alors, la restriction de $\rho \underline{\text { ab }}$ à l'inertie coïncide avec le composé de $\chi_{1}: G_{K} \rightarrow K_{1}^{*}$ avec $\eta_{1}^{\text {ab }}$.

\section{Etude globale.}

2.0. Soit $\overline{\mathbf{Q}}$ la clôture algébrique de $\mathbf{Q}$ dans le corps des nombres complexes $\mathbf{C}$ et $K \subset \overline{\mathbf{Q}}$ un corps de nombres. Notons $G_{\mathbf{Q}}$ et $G_{K}$ les groupes de Galois de $\overline{\mathbf{Q}} / \mathbf{Q}$ et de $\overline{\mathbf{Q}} / K$ respectivement. Pour tout idéal premier $\overline{\mathcal{Q}}$ de $\overline{\mathbf{Q}}$, notons $D_{\overline{\mathcal{Q}}}$ le sous-groupe de décomposition en $\overline{\mathcal{Q}}$. On se donne d'autre part une clôture algébrique $\overline{\mathbf{Q}_{p}}$ de $\mathbf{Q}_{p}$, et on note $C_{p}$ la complétion de $\overline{\mathbf{Q}_{p}}$.

Si $E, E^{\prime}$, et $E^{\prime \prime}$ sont trois corps, avec $E \subset E^{\prime}$ et $E \subset E^{\prime \prime}$, on note $\mathrm{P}_{E}\left(E^{\prime}, E^{\prime \prime}\right)$ l'ensemble des $E$-plongements de $E^{\prime}$ dans $E^{\prime \prime}$.

\subsection{Groupes à un paramètre de Hodge-Tate.}

Soit $H$ un groupe algébrique affine sur $\mathbf{Q}_{p}$ dont la composante neutre $H^{0}$ est un 
groupe réductif. Soit $\rho: G_{K} \rightarrow H\left(\mathbf{Q}_{p}\right)$ une représentation $p$-adique dont les restrictions à tous les sous-groupes de décomposition $D \bar{\wp}$ en les premiers $\bar{\wp}$ de $\overline{\mathbf{Q}}$ au dessus de $p$ sont de Hodge-Tate.

Pour tout $\tau \in \mathrm{P}_{\mathbf{Q}}\left(\overline{\mathbf{Q}}, \overline{\mathbf{Q}_{p}}\right)$, on note $D_{\tau} \subset G_{K}$ le sous-groupe de décomposition en la place de $\overline{\mathbf{Q}}$ définie par $\tau$. Donc, $D_{\tau}$ s'identifie, via $\tau$, au groupe de Galois $\left.\operatorname{Gal}\left(\overline{\mathbf{Q}_{p}} / \widetilde{\tau(K}\right)\right)$ du complété de l'image de $K$ par $\tau$ dans $\overline{\mathbf{Q}_{p}}$. On désigne par $\mu_{\mathrm{HT}, \tau}$ le sous-groupe à un paramètre de $H^{0}$ défini par la décomposition de Hodge-Tate de la restriction de $\rho$ à $D_{\tau} \simeq \operatorname{Gal}\left(\overline{\mathbf{Q}_{p}} / \tau(\widehat{(K})\right)$. On note $\mathcal{C}\left(\mu_{\mathrm{HT}, \tau}\right)$ son image dans $W(R) \backslash Y$ (cf 0.1., on note encore $R$ le système de racines de $\left.H^{0}\right)$ et $\pi_{1}\left(\mu_{\mathrm{HT}, \tau}\right)$ son image dans $\pi_{1}\left(H^{0}\right)$.

On a ([Se78], 1.5.), pour $\sigma \in G_{\mathbf{Q}_{p}}$ :

$$
\text { (1) } \mu_{\mathrm{HT}, \sigma \circ \tau}={ }^{\sigma}\left(\mu_{\mathrm{HT}, \tau}\right) \text {. }
$$

De plus, il est clair que, par fonctorialité, on a pour $\gamma \in G_{K}$ :

$$
\text { (2) } \mu_{\mathrm{HT}, \tau \circ \gamma}=\operatorname{int}\left(\rho\left(\gamma^{-1}\right)\right)\left(\mu_{\mathrm{HT}, \tau}\right) \text {. }
$$

Soit $K^{\prime}$ le corps fixé par le noyau de $\rho$ composé avec $H\left(\mathbf{Q}_{p}\right) \rightarrow\left(H / H^{0}\right)\left(\mathbf{Q}_{p}\right)$. On en déduit de (1) et de (2) que $\mathcal{C}\left(\mu_{\mathrm{HT}, \tau}\right)$ ne dépend que de la restriction de $\tau$ à $K^{\prime}$ et que, si $\sigma \in G_{\mathbf{Q}_{p}}:$

$$
\mathcal{C}\left(\mu_{\mathrm{HT}, \sigma \circ \tau}\right)={ }^{\sigma} \mathcal{C}\left(\mu_{\mathrm{HT}, \tau}\right), \pi_{1}\left(\mu_{\mathrm{HT}, \sigma \circ \tau}\right)={ }^{\sigma} \pi_{1}\left(\mu_{\mathrm{HT}, \tau}\right) .
$$

Soit $T_{K^{\prime}}$ le tore sur $\mathbf{Q}$ restriction des scalaires à la Weil de $K^{\prime}$ à $\mathbf{Q}$ du groupe multiplicatif. Son groupe des cocaractèrs $Y\left(\left(T_{K^{\prime}}\right) \overline{\mathbf{Q}_{p}}\right)$ s'identifie donc au groupe abélien libre de base l'ensemble $\mathrm{P}_{\mathbf{Q}}\left(K^{\prime}, \overline{\mathbf{Q}_{p}}\right)$. Il résulte de la formule ci-dessus donnant l'action de $G_{\mathbf{Q}_{p}}$ sur les $\pi_{1}\left(\mu_{\mathrm{HT}, \tau}\right)$ que l'on a un morphisme $g_{\mathrm{HT}} \operatorname{de} Y\left(\left(T_{K^{\prime}}\right) \overline{\mathbf{Q}_{p}}\right)$ dans $\pi_{1}\left(H_{\overline{\mathbf{Q}_{p}}}\right)$ qui commute à l'action de $G_{\mathbf{Q}_{p}}$. On en déduit un morphisme $c f$ 0.3. :

$$
\text { (3) : } \mathrm{B}\left(g_{\mathrm{HT}}\right): T_{K^{\prime}}\left(\mathbf{Q}_{p}\right)=\left(K^{\prime} \otimes \mathbf{Q}_{p}\right)^{*} \rightarrow H_{\mathrm{ab}}^{0}\left(\mathbf{Q}_{p}, H^{0}\right) .
$$

2.2. On suppose de plus que $H$ est connexe $\left(\mathrm{K}=\mathrm{K}^{\prime}\right)$ et que les restrictions de $\rho$ à tous les sous-groupes de décomposition $D_{\overline{\mathcal{Q}}}$, sont semi-stables. Pour $\overline{\mathcal{Q}}$ ne divisant pas $p$, ceci signifie que l'action du sous-groupe d'inertie de $D \overline{\mathcal{Q}}$ est unipotente, pour $\overline{\mathcal{Q}}$ divisant $p$, c'est au sens de [Fo94]. On note $\rho \underline{\text { ab }}$ le composé de $\rho$ avec $H\left(\mathbf{Q}_{p}\right) \rightarrow H_{\mathrm{ab}}^{0}\left(\mathbf{Q}_{p}, H\right)$.

Proposition. Le morphisme $\rho \frac{\mathrm{ab}}{\underline{a}}: G_{K} \rightarrow H_{\mathrm{ab}}^{0}\left(\mathbf{Q}_{p}, H\right)$ est non ramifié en dehors des idéaux premiers de $K$ qui sont au dessus de $p$ (et de l'infini).

Démonstration. Il suffit de prouver qu'un élément unipotent $u$ de $H\left(\mathbf{Q}_{p}\right)$ est dans l'image de $S_{\mathrm{sc}}\left(\mathbf{Q}_{p}\right), S_{\mathrm{sc}}$ revêtement universel de $S=[H, H]$. Un tel $u$ a une image triviale dans $H^{\mathrm{ab}}\left(\mathbf{Q}_{p}\right)=(H / S)\left(\mathbf{Q}_{p}\right)$. Il existe donc $g \in S_{\mathrm{sc}}\left(\overline{\mathbf{Q}_{p}}\right)$ tel que $\pi(g)=u$, $\pi$ désignant le morphisme $S_{\mathrm{sc}} \rightarrow S$. Soit $g=u^{\prime} s^{\prime}$ la décomposition de $g$ en le produit d'un unipotent et d'un semi-simple commutant. Alors $s^{\prime} \in \operatorname{Ker}(\pi)$. On en déduit facilement que $u^{\prime}$ est l'unique unipotent d'image $u$ dans $S\left(\mathbf{Q}_{p}\right) ; u^{\prime}$ est donc rationnel sur $\mathbf{Q}_{p}$, ce qui prouve la proposition. 
2.3. On déduit de 1.3. la proposition suivante :

Proposition. Soit $G_{K, p}$ le groupe de Galois de l'extension maximale de $K$ qui est non ramifiée en dehors de $p$ et de l'infini et soit $G_{K, p}^{\mathrm{ab}}$ son plus grand quotient abélien. Soit $r$ l'application de réciprocité : $U_{K \otimes \mathbf{Q}_{p}} \rightarrow G_{K, p}^{\mathrm{ab}}$, le groupe $U_{K \otimes \mathbf{Q}_{p}}$ désignant le produit des groupes des unités des différents complétés de $K$ aux premiers aux dessus de p. Soit $\mathrm{B}\left(g_{\mathrm{HT}}\right)$ comme dans (3) de 2.1.. Alors, le diagramme suivant commute:

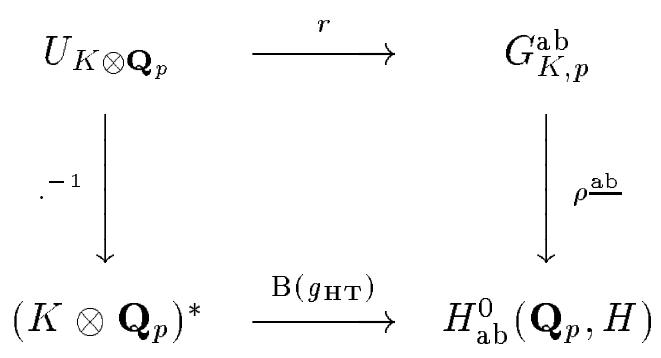

.$^{-1}$ désignant $u \mapsto u^{-1}$.

2.4. Corollaire. On suppose $K$ non ramifié au dessus de $p$. Soit $h$ un groupe à un paramètre de $H$ qui est défini sur $\mathbf{Q}_{p}$. On suppose que l'image de $g_{\mathrm{HT}}$ contient $\pi_{1}(h)$. Alors $\rho \underline{\mathrm{ab}}\left(G_{K}\right)$ contient l'image de $h\left(\mathbf{Z}_{p}^{*}\right)$ dans $H_{\mathrm{ab}}^{\mathrm{O}}\left(\mathbf{Q}_{p}, H\right)$.

2.5. Démonstration. Soit $y \in Y\left(\left(T_{K}\right) \overline{\mathbf{Q}_{p}}\right)$ tel que $g_{\mathrm{HT}}(y)=\pi_{1}(h)$. Le fixateur de $y$ dans $G_{\mathbf{Q}_{p}}$ est un sous-groupe ouvert correspondant à une extension finie de $\mathbf{Q}_{p}$ qui est non ramifiée, puisque $K$ est non ramifié au dessus de $p$; désignons cette extension par $E$. Le groupe à un paramètre $y$ définit un morphisme sur $\mathbf{Q}_{p}$ de $T_{E}$ dans $\left(T_{K}\right)_{\mathbf{Q}_{p}}$, et donc un morphisme du groupe des unités $U_{E}$ de $E$ dans $U_{K \otimes \mathbf{Q}_{p}}$. Le diagramme suivant est commutatif :

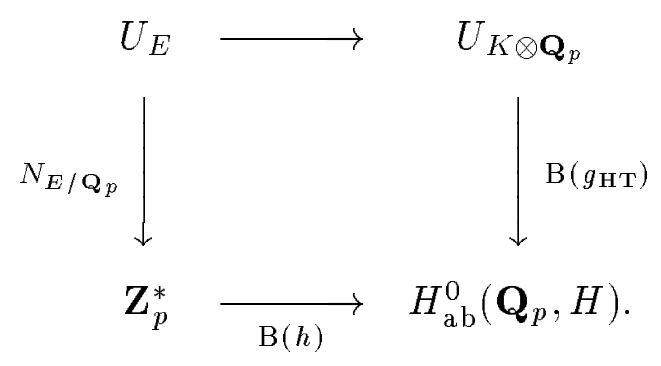

La norme $N_{E / \mathbf{Q}_{p}}: U_{E} \rightarrow \mathbf{Z}_{p}^{*}$ est surjective, puisque $E$ est non ramifié sur $\mathbf{Q}_{p}$. Il résulte de la proposition précédente que l'image de Galois dans $H_{\mathrm{ab}}^{0}\left(\mathbf{Q}_{p}, H\right)$ contient $\mathrm{B}\left(g_{\mathrm{HT}}\right)\left(U_{K \otimes \mathbf{Q}_{p}}\right)$. Le corollaire en résulte.

2.6. Le tore $T_{K, \mathrm{~cm}}$. 
Soient $K_{\mathrm{tr}}$ et $K_{\mathrm{cm}}$ les plus grands sous-corps totalement réel et CM contenus dans $K$. $\mathrm{Si}$, comme dans [Se68] chap. 2., $T_{\mathcal{M}}$ est le tore sur $\mathbf{Q}$ associé à un modulus $\mathcal{M}$ du corps de nombres $K$, on note $T_{K, \mathrm{~cm}}$ le tore $T_{\mathcal{M}}$ pour un modulus $\mathcal{M}$ suffisamment grand pour que le noyau de $T_{K} \rightarrow T_{K, \mathrm{~cm}}$ soit un tore (autrement dit soit connexe). Il est clair que cette définition ne dépend pas du choix d'un tel modulus $\mathcal{M}$. Le groupe des cocaractères $Y\left(T_{K}\right)$ sur $\overline{\mathbf{Q}}$ de $T_{K}$ est le groupe abélien libre $\mathbf{Z}\left[P_{\mathbf{Q}}(K, \overline{\mathbf{Q}})\right]$ de base $P_{\mathbf{Q}}(K, \overline{\mathbf{Q}})$. Le groupe $Y\left(T_{K, \mathrm{~cm}}\right)$ des cocaractères sur $\overline{\mathbf{Q}}$ de $T_{K, \mathrm{~cm}}$ s'identifie donc à un quotient de $\mathbf{Z}\left[P_{\mathbf{Q}}(K, \overline{\mathbf{Q}})\right]$. Il est décrit de la manière suivante :

2.6. Proposition. Si $K_{\mathrm{tr}}=K_{\mathrm{cm}}, Y\left(T_{K, \mathrm{~cm}}\right)$ s'identifie au quotient de $\mathbf{Z}\left[P_{\mathbf{Q}}(K, \overline{\mathbf{Q}})\right]$ par le morphisme d'augmentation $\left(\sum n_{\tau} \tau \mapsto \sum n_{\tau}\right)$. Si $K_{\mathrm{tr}} \neq K_{\mathrm{cm}}, Y\left(T_{K, \mathrm{~cm}}\right)$ s'identifie au quotient de $\mathbf{Z}\left[P_{\mathbf{Q}}\left(K_{\mathrm{cm}}, \overline{\mathbf{Q}}\right)\right]$ par les relations (redondantes) :

$$
\text { (4) : } \tau_{1}+c \tau_{1}=\ldots=\tau_{s}+c \tau_{s},
$$

où $\tau_{1}, \ldots, \tau_{s}$ sont les différents plongements de $K_{\mathrm{cm}}$ dans $\overline{\mathbf{Q}}$ et c la conjugaison complexe de $K_{\mathrm{cm}}$.

Démonstration. C'est vrai après tensorisation avec $\mathbf{Q}$ d'après [Se68] chap. 23 . La proposition en résulte car les quotients décrits dans la proposition sont sans torsion.

\section{7.}

2.7.1. Soit $\mathcal{M}_{\mathrm{dR}, K}$ la catégorie des motifs pour les cycles de Hodge absolus qui sont définis sur $K$ ([DeMi82]) et de Rham au sens de [B194]. Rappelons qu'un cycle de Hodge absolu $t$ défini sur $K$ est de Rham si pour tout plongement $\iota$ de $\overline{\mathbf{Q}}$ dans $\overline{\mathbf{Q}_{p}}$ les composantes de Rham et étales $p$-adiques de $\iota(t)$ se correspondent dans le théorème de comparaison $p$-adique. Les cycles algébriques et les cycles de Hodge des variétés abéliennes sont de Rham ([Ts98] et [B194]).

Soit $M$ de $\mathcal{M}_{\mathrm{dR}, K}$. On suppose que $M$ est pur de poids $m$. Soit $h_{\mathrm{B}}(M)$ la réalisation Betti de $M$.

Soit $H_{\mathbf{Q}}$ un sous-groupe réductif connexe de $\mathrm{GL}_{h_{\mathrm{B}}(M)}$ qui est défini par des tenseurs qui sont la réalisation Betti de cycles de Hodge absolus définis sur $K$ et qui sont de Rham (dans la sous-catégorie tannakienne de $\mathcal{M}_{\mathrm{dR}, K}$ engendrée par $M$ et le motif de Tate). La réalisation étale $p$-adique de $M$ fournit alors une représentation $p$-adique $\rho: G_{K} \rightarrow H\left(\mathbf{Q}_{p}\right)$ (nous notons $H$ le groupe sur $\mathbf{Q}_{p}$ obtenu à partir de $H$ par extension des scalaires de $\mathbf{Q}$ à $\left.\mathbf{Q}_{p}\right)$.

Soit $\mu_{\mathrm{H}}$ le groupe à un paramètre de $H_{\mathbf{C}}$ défini par la théorie de Hodge complexe (donc $\mu_{\mathrm{H}}$ a pour poids $-i$ sur $\left.H^{i, j}\right)$. Notons $\pi_{1}\left(\mu_{\mathrm{H}}\right)$ sa classe dans $\pi_{1}\left(H_{\overline{\mathbf{Q}}}\right)$.

Soit $w$ le groupe à un paramètre de $H_{\mathbf{Q}}$ donnant la graduation par le poids ([DeMi82]). Comme le motif $M$ est pur de poids $m$, le groupe à un paramètre $w$ est la puissance $m$-ième des homothéties. Si $c$ est la conjugaison complexe, on a : $\mu_{\mathrm{H}}+c\left(\mu_{\mathrm{H}}\right)=-w$. D'où :

$$
\text { (5) : } \pi_{1}\left(\mu_{\mathrm{H}}\right)+c\left(\pi_{1}\left(\mu_{\mathrm{H}}\right)\right)=-\pi_{1}(w)
$$

2.7.2. Proposition. $\pi_{1}\left(\mu_{\mathrm{H}}\right)$ est fixe par $G_{K}$. Soit alors $g_{\mathrm{H}}$ l'unique morphisme, commutant à l'action de $G_{\mathbf{Q}}$, du groupe des cocaractères $Y\left(T_{K}\right)$ de $\left(T_{K}\right) \overline{\mathbf{Q}}$ dans $\pi_{1}\left(H_{\overline{\mathbf{Q}}}\right)$ 
qui envoie l'inclusion de $K$ dans $\overline{\mathbf{Q}}$ sur $\pi_{1}\left(\mu_{\mathrm{H}}\right)$. Alors, si $K_{\mathrm{tr}} \neq K_{\mathrm{cm}}$, le morphisme $g_{\mathrm{H}}$ se factorise à travers $Y\left(T_{K, \mathrm{~cm}}\right)$.

2.7.3. Démonstration. Soit $\operatorname{Rep}_{\mathbf{Q}}\left(H_{\mathbf{Q}}\right)$ la catégorie des représentations linéaires du groupe algébrique $H_{\mathbf{Q}}$ dans les $\mathbf{Q}$-espaces vectoriels de dimensions finies. Comme $H_{\mathbf{Q}}$ est défini par des tenseurs qui sont les réalisations Betti de cycles de Hodge absolus qui sont définis sur $K$ et qui sont de Rham, une représentation linéaire de $H_{\mathbf{Q}}$ dans un Q-espace vectoriel de dimension finie définit un motif de $\mathcal{M}_{\mathrm{dR}, K}$ et on dispose d'un foncteur naturel de $\operatorname{Rep}_{\mathbf{Q}}\left(H_{\mathbf{Q}}\right)$ dans $\mathcal{M}_{\mathrm{dR}, K}$. La réalisation de Hodge $\operatorname{gr}\left(H_{\mathrm{dR}}\right)$, graduée pour la filtration de Hodge de la réalisation de Rham, définit un foncteur fibre de $\mathcal{M}_{\mathrm{dR}, K}$ et donc de $\operatorname{Rep}_{\mathbf{Q}}\left(H_{\mathbf{Q}}\right)$. On note ce dernier $\omega_{\mathrm{H}}$. Un point du torseur des $\otimes$-isomorphismes de $\omega_{\mathrm{H}}$ sur le foncteur fibre réalisation de Betti, envoie le groupe à un paramètre défini par la graduation de $\omega_{\mathrm{H}}$ sur un conjugué par automorphismes intérieurs de $-\mu_{\mathrm{H}}$. Comme la graduation de $\omega_{\mathrm{H}}$ est définie sur $K$, cela prouve que la classe de conjugaison de $\mu_{\mathrm{H}}$ dans $H_{\overline{\mathbf{Q}}}$ est définie sur $K$ et $\pi_{1}\left(\mu_{\mathrm{H}}\right)$ est bien fixe par $G_{K}$.

Il résulte de (5) que l'on a pour tout $\tau \in G_{\mathbf{Q}}$ :

$$
\tau\left(\pi_{1}\left(\mu_{\mathrm{H}}\right)\right)+(\tau \circ c)\left(\pi_{1}\left(\mu_{\mathrm{H}}\right)\right)=-\pi_{1}(w) .
$$

On voit avec (4) qu'il reste à prouver que, pour tout $\tau \in G_{\mathbf{Q}}$, on a :

$$
(\tau \circ c)\left(\pi_{1}\left(\mu_{\mathrm{H}}\right)\right)=(c \circ \tau)\left(\pi_{1}\left(\mu_{\mathrm{H}}\right)\right) .
$$

Le sous-groupe de $G_{\mathbf{Q}}$ engendré par les $\tau \circ c \circ \tau^{-1} \circ c$ est le groupe de Galois de la plus grande extension $\mathrm{CM}$ de $\mathbf{Q}$ contenue dans $\overline{\mathbf{Q}}$ ([Se68] chap. 2 3). On la note $\mathbf{Q}_{\mathrm{CM}}$. Il s'agit donc de prouver que $G_{\mathbf{Q}_{\mathrm{CM}}}$ fixe $\pi_{1}\left(\mu_{\mathrm{H}}\right)$. Il suffit pour cela de prouver que la classe de conjugaison de $\mu_{\mathrm{H}}$ dans $H_{\overline{\mathbf{Q}}}$ est définie sur $\mathbf{Q}_{\mathrm{CM}}$.

Soit $q$ une forme de Weil de $M$ qui est définie sur $K\left([\mathrm{DeMi} 82]\right.$ Th. 6.7.) et $H^{\prime} \subset H_{\mathbf{Q}}$ le sous-groupe de $H$ qui fixe $q: M \otimes M \rightarrow \mathbf{Q}(-m)$. Le groupe à un paramètre $\mu_{\mathrm{B}}$ est à valeurs dans la composante neutre $H^{\prime 0}$ de $H^{\prime}$. Pour prouver que la classe de conjugaison de $\mu_{\mathrm{H}}$ dans $\left(H_{\mathbf{Q}}\right)_{\mathbf{Q}}$ est définie sur $\mathbf{Q}_{\mathrm{CM}}$, il suffit de prouver qu'il en est ainsi de la classe de conjugaison de $\mu_{\mathrm{H}}$ dans $\left({H^{\prime}}^{0}\right)_{\overline{\mathbf{Q}}}$. Soit $\left(X^{\prime}, Y^{\prime}, R^{\prime}, \alpha^{\prime} \rightarrow \alpha^{\prime \vee}, B^{\prime}\right)$ le système de racines épinglé associé au quotient de la composante neutre de $H^{\prime}$ par son radical unipotent. Comme le quotient de ${H^{\prime}}^{0}$ par l'image du groupe à un paramètre des poids $w$ est une forme intérieure de sa forme compacte ([DeMi82] Re. 5.17.), la conjugaison complexe $c$ agit comme l'involution fondamentale sur $R^{\prime}$ et comme la multiplication par -1 sur le groupe de caractères du quotient de la composante neutre du centre de $H^{\prime}$ par l'image de $w$. On voit alors que l'action de $c \operatorname{sur}\left(X^{\prime}, Y^{\prime}, R^{\prime}, \alpha^{\prime} \rightarrow \alpha^{\prime \vee}, B^{\prime}\right)$ se fait par un automorphisme qui commute à l'action de $G_{\mathbf{Q}}$. On en déduit facilement que l'action du groupe de Galois absolu de $G_{\mathbf{Q}_{\mathrm{CM}}}$ sur le système de racines épinglé de $H^{\prime}$ est trivial, ce qui prouve que $\mathcal{C}\left(\mu_{\mathrm{H}}\right)$ est bien fixe par $G_{\mathbf{Q}_{\mathrm{CM}}}$ et achève de prouver la propostion.

2.7.4. Le morphisme $g_{\mathrm{HT}}$ se déduit de $g_{\mathrm{H}}$ par extension des scalaires de $\mathbf{Q}$ à $\mathbf{Q}_{p}$. Plus précisément :

Proposition. Soit $\iota_{0}$ un plongement de $\overline{\mathbf{Q}}$ dans $\overline{\mathbf{Q}_{p}}$. Pour tout plongement $\tau$ de $K$ dans $\overline{\mathbf{Q}}$, on a : 


$$
\iota_{0}\left(g_{\mathrm{H}}(\tau)\right)=g_{\mathrm{HT}}\left(\iota_{0} \circ \tau\right) .
$$

De plus, pour tout plongement $\iota$ de $\overline{\mathbf{Q}}$ dans $\overline{\mathbf{Q}_{p}}$, on a :

$$
\text { (6) : } \pi_{1}\left(\mu_{\mathrm{HT}, \iota}\right)+\pi_{1}\left(\mu_{\mathrm{HT}, \iota \circ \mathrm{C}}\right)=-\pi_{1}(w) \text {. }
$$

Démonstration. $\iota_{0}\left(g_{\mathrm{H}}(\tau)\right)$ est l'image dans $\pi_{1}(H)$ de $\left(\iota_{0} \circ \tau\right)\left(\mathcal{C}\left(\mu_{\mathrm{H}}\right)\right)$. D'autre part :

$$
g_{\mathrm{HT}}\left(\iota_{0} \circ \tau\right)=\pi_{1}\left(\mu_{\mathrm{HT}, \iota_{0} \circ \tau}\right) .
$$

On voit que pour prouver la première partie de la proposition, il suffit de voir que, pour tout plongement $\iota$ de $\overline{\mathbf{Q}}$ dans $\overline{\mathbf{Q}_{p}}$, on a :

$$
\iota\left(\pi_{1}\left(\mu_{\mathrm{H}}\right)\right)=\pi_{1}\left(\mu_{\mathrm{HT}, \iota}\right) .
$$

On a : $\iota\left(\mathcal{C}\left(\mu_{\mathrm{H}}\right)\right)=\mathcal{C}\left(\mu_{\mathrm{HT}, \iota}\right)$ : c'est un conséquence du fait que les cycles de Hodge absolus qui définissent $M$ et $H_{\mathbf{Q}}$ sont compatibles aux théorèmes de comparaison $p$-adiques ([Wi88]). La première partie de la proposition en résulte.

La seconde partie en résulte alors en appliquant à (5) le plongement $\iota_{0}$.

2.7.5. Soit $A$ une variété abélienne sur le corps de nombres $K$. Soit $\rho_{p}: G_{K} \rightarrow H\left(\mathbf{Q}_{p}\right)$ la représentation $p$-adique associée au module de Tate de $A, H$ désignant l'adhérence de Zariski de l'image de $G_{K}$ dans $\mathrm{Gl}_{V_{p}(A)}$. On sait que le groupe algébrique $H$ contient les homothéties ([Bo80]). On a :

Corollaire. On suppose que $K$ est non ramifié au dessus de $p$. On suppose que $A$ a réduction semi-stable en tous les idéaux premiers de $K$ au dessus de $p$. On suppose, soit que $A$ vérifie la conjecture de Mumford-Tate, soit que la dimension de A est inférieure ou égale à 4. Alors $\rho \frac{\mathrm{ab}}{p}\left(G_{K}\right) \subset H_{\mathrm{ab}}^{0}\left(\mathbf{Q}_{p}, H^{0}\right)$ contient l'image des homothéties $w\left(\mathbf{Z}_{p}^{*}\right)$ par le morphisme $H^{0}\left(\mathbf{Q}_{p}\right) \rightarrow H_{\mathrm{ab}}^{0}\left(\mathbf{Q}_{p}, H^{0}\right)$.

Démonstration. Comme $A$ a réduction semi-stable en les premiers au dessus de $p$, il existe une extension finie $K^{\prime}$ de $K$, qui est non ramifiée au dessus de $p$ et telle que $A$ a réduction semi-stable en tous les premiers de $K^{\prime}$ (on peut prendre pour $K^{\prime}$ l'extension qui rend rationnels les points d'ordre $\ell_{1} \ell_{2}$ pour $\ell_{1}$ et $\ell_{2}$ premiers distincts $\geq 3$ et $\neq p$ ). Comme $A$ a réduction semi-stable sur $K$, l'adhérence de Zariski de l'image des sous-groupes d'inertie en les premiers de $K$ au dessus de $p$ est connexe (1.2.5.). Il en résulte que l'on peut supposer de plus que l'adhérence de Zariski de $\rho_{p}\left(G_{K^{\prime}}\right)$ est connexe. Clairement, il suffit de prouver le corollaire après avoir remplacé $K$ par $K^{\prime}$.

D'après le corollaire 2.4., il suffit pour cela de prouver que l'image de $g_{\mathrm{HT}}$ contient $\pi_{1}(w)$.

Si $A$ vérifie la conjecture de Mumford et Tate, le groupe $H$ s'identifie au groupe de Mumford-Tate MT tensorisé avec $\mathbf{Q}_{p}$. Le groupe de Mumford-Tate est défini comme le sous-groupe de $\mathrm{Gl}_{H_{\mathrm{B}}^{1}(A)} \times \mathbf{G}_{\mathrm{m}}$ fixant les composantes Betti des cycles de Hodge absolus de sous-catégorie de $\mathcal{M}_{\mathrm{dR}, K}$ engendré par le motif associé à $A$ ([De82] 3). Comme ces cycles sont de Rham, l'ǵalité (6) de 2.7.4. entraîne que l'on a $\pi_{1}(w)=-g_{\mathrm{HT}}(\iota+\iota \circ c)$ pour tout plongement $\iota$ de $\overline{\mathbf{Q}}$ dans $\overline{\mathbf{Q}_{p}}$. Cela prouve le corollaire. 
Si $A$ est de dimension $\leq 4, A$ vérifie la conjecture de Mumford et Tate, sauf peut-être si $A$ est de dimension 4 et que l'adhérence de Zariski de l'image de Galois $H$ est une forme de l'image de :

$$
\mathrm{G}_{\mathrm{m}} \times \mathrm{SL}_{2} \times \mathrm{SL}_{2} \times \mathrm{SL}_{2}
$$

dans la représentation stand $\otimes$ stand $\otimes$ stand, stand désignant la représentation standard de $\mathrm{SL}_{2}$ ([MoZa95]), et $\mathrm{G}_{\mathrm{m}}$ agissant par les homothéties (alors que le groupe de Mumford-Tate serait le groupe des similitudes simplectiques). Soit $\iota$ un plongement de $\overline{\mathbf{Q}}$ dans $\overline{\mathbf{Q}_{p}}$. Il est facile de voir que comme $\mu_{\mathrm{HT}, \iota}$ a pour poids 0 et $1, \mu_{\mathrm{HT}, \iota}(\lambda)$ est, à permutation des facteurs $\mathrm{SL}_{2}$ près, conjugué à :

$$
\left(\begin{array}{ll}
\lambda & 0 \\
0 & 1
\end{array}\right) \otimes \mathrm{id} \otimes \mathrm{id}
$$

Cette matrice est conjuguée dans $H$ à la matrice :

$$
\left(\begin{array}{ll}
1 & 0 \\
0 & \lambda
\end{array}\right) \otimes \mathrm{id} \otimes \mathrm{id}
$$

Comme le produit de ces deux matrices agit comme l'homothétie de rapport $\lambda$, on en déduit que $\pi_{1}(w)=-2 g_{\mathrm{HT}}(\iota)$, ce qui prouve le corollaire aussi dans ce cas.

[B194] Don BLASIUS A p-adic property of Hodge classes on abelian varieties. Motives (Seattle, WA, 1991), 293-308, Proc. Sympos. Pure Math., 55, Part 2, Amer. Math. Soc., Providence, RI, 1994.

[Bo80] F.A. BOGOMOLOV. Sur l'algébricité des représentations p-adiques. C.R. Acad. Sci.Paris, 290 (1980), 701-703.

[Bo98] Mikhail BOROVOI. Abelian Galois cohomology of reductive groups. Mem. Amer. Math. Soc. 132 (1998), no. 626.

[CG] Jean-Pierre SERRE. Cohomologie Galoisienne. Lecture Notes in Mathematics 5. Cohomologie galoisienne. 5-ième edition. Lecture Notes in Mathematics, 5. SpringerVerlag, Berlin, 1994.

[CoFo99] Pierre COLMEZ et Jean-Marc FONTAINE. Construction de représentations $p$-adiques semi-stables. Preprint 99. A paraître à Inventiones.

[De79] Pierre DELIGNE. Variétés de Shimura: interprétation modulaire, et techniques de construction de modèles canoniques. Automorphic forms, representations and $L$-functions. Proc. Sympos. Pure Math., Oregon State Univ., Corvallis, Ore., 1977, Part 2, pp. 247-289, Proc. Sympos. Pure Math., XXXIII, Amer. Math. Soc., Providence, R.I., 1979.

[De82] Pierre DELIGNE. Hodge cycles on abelian varieties. Dans Deligne, Milne, Ogus, Shih, Hodge cycles, motives, and Shimura varieties. Lecture Notes in Mathematics, 900. Springer-Verlag, Berlin-New York, 1982. 
[DeMi82] Pierre DELIGNE, James S. MILNE. Tannakian categories. Dans Deligne, Milne, Ogus, Shih, Hodge cycles, motives, and Shimura varieties. Lecture Notes in Mathematics, 900. Springer-Verlag, Berlin-New York, 1982.

[Fo94] Jean-Marc FONTAINE. Représentations $p$-adiques semi-stables. Périodes $p$ adiques (Bures-sur-Yvette, 1988). Astérisque No. 223 , (1994), p. 113-184.

[MoZa95] Ben MOONEN, Yuri ZAHRIN. Hodge classes and Tate classes on simple abelian fourfolds. Duke Math. J. 77 (1995), no. 3, 553-581.

[Sa95] Takeshi SAITO. The sign of the functional equation of the $L$-function of an orthogonal motive. Invent. Math. 120 (1995), no. 1, p. 119-142.

[Se68] Jean-Pierre SERRE. Abelian $l$-adic representations and elliptic curves. With the collaboration of Willem Kuyk and John Labute. Revised reprint of the 1968 original. Research Notes in Mathematics, 7. A K Peters, Ltd., Wellesley, MA, 1998.

[Se78] Jean-Pierre SERRE. Groupes algébriques associés aux modules de Hodge-Tate. Dans: Journées de Géométrie Algébrique de Rennes. Astérisque 65. 1979.

[St65] R STEINBERG. Regular elements of semisimple algebraic groups. Publications IHES, 25 (1965).

[Ts98] Takeshi TSUJI. $p$-adic Hodge theory in the semi-stable reduction case. Proceedings of the International Congress of Mathematicians, Vol. II (Berlin, 1998). Doc. Math. 1998, Extra Vol. II, 207-216.

[Wi88] Jean-Pierre WINTENBERGER. Motifs et points d'ordre finis des variétés abéliennes. Séminaire de Théorie des Nombres. Paris 1986-87. Edited by Catherine Goldstein. Progress in Mathematics, Birkhauser, 1988.

[Wi95] Jean-Pierre WINTENBERGER. Relèvement selon une isogénie de systèmes $l$-adiques de représentations galoisiennes associées aux motifs. Invent. Math. 120 (1995), no. 2, p. 215-240.

[Wi97] Jean-Pierre WINTENBERGER. Propriétés du groupe tannakien des structures de Hodge $p$-adiques et torseur entre cohomologies cristalline et étale. Ann. Inst. Fourier (Grenoble) 47 (1997), no. 5, p. 1289-1334.

Jean-Pierre Wintenberger

Université Louis Pasteur

Département de Mathématique

7, rue René Descartes

67084 Strasbourg

France

Courrier électronique : wintenb@math.u-strasbg.fr 